\title{
Experimental verification of agglomeration effects in infrared spectra on micron-sized particles
}

\author{
Akemi Tamanai ${ }^{1}$, Jochen Vogt ${ }^{1}$, Christian Huck ${ }^{1}$, Uwe Mick $^{2}$, Sören Zimmermann ${ }^{3}$, Ryo Tazaki ${ }^{4}$, \\ Harald Mutschke ${ }^{5}$, and Annemarie Pucci ${ }^{1}$

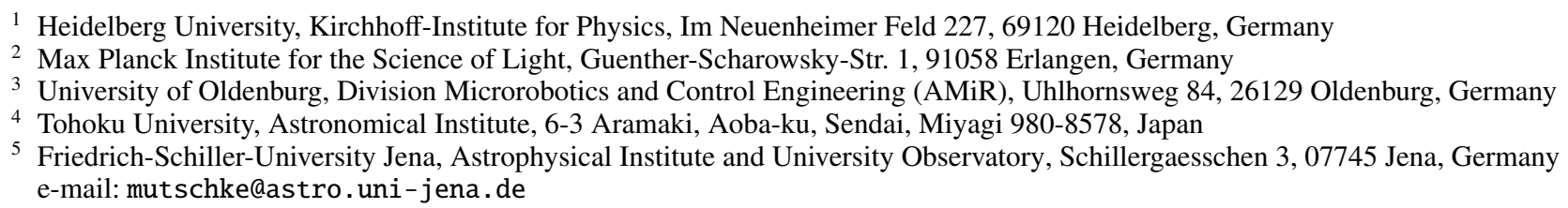

Received 28 March 2018 / Accepted 26 August 2018

\begin{abstract}
Context. Detailed analysis of observed infrared (IR) dust emission spectra is often performed in order to derive information about mineralogy, particle size, and temperature of the dust. However, the IR bands are also influenced by agglomeration of the dust particles. Light scattering theory simulating agglomeration and growth effects is especially challenged by the consideration of highly absorbing particles.

Aims. To clarify the influence of agglomeration on the diagnostic phonon bands of amorphous $\mathrm{SiO}_{2}$ particles, we experimentally measure the extinction spectra of systematically arranged particle configurations and compare the measured spectra with the spectra obtained from different theoretical approaches.

Methods. We construct artificial particle agglomerates by means of the dedicated robotic manipulation (DRM) technique. IR microspectroscopic extinction measurements of these arranged particles are performed at the French National Synchrotron Facility, SOLEIL, in the mid-IR region considering polarization effects. The theoretical approaches applied are the discrete dipole approximation (DDA) as well as T-matrix and finite-difference time-domain methods.

Results. In both the experimental spectra and the theoretical calculations, we find that the $\mathrm{Si}-\mathrm{O}$ stretching vibration band at about $9 \mu \mathrm{m}$ is clearly broadened on the long-wavelength side by the agglomeration of particles. This is mainly caused by the radiation components, which are polarized in directions in which the agglomerate is extended, while the extinction band profile of the component polarized perpendicular to the long axis of an elongated agglomerate is close to the spectrum of the single sphere. All of the theoretical simulations predict these effects in qualitatively good agreement.

Conclusions. Our comparative study of the experimentally measured and theoretically calculated IR extinction spectra of well-defined agglomerate structures makes obvious how the various particle arrangements in small clusters might contribute to average spectra of dust. Therefore the study might help to improve the precision of light scattering calculations as well as their specific applicability.
\end{abstract}

Key words. infrared: general - methods: laboratory: solid state - methods: data analysis - polarization - circumstellar matter planetary systems

\section{Introduction}

Nano- and micron-sized dust grains effectively absorb and scatter incident radiation, and thus control the thermal balance as well as dynamical processes in astronomical environments. Absorption, emission, and scattering of light strongly depend on the physical and chemical properties of the dust grains. This is particularly true for the infrared (IR) spectral range where dust absorbs and emits preferentially in bands with characteristic frequencies, which are defined by the lattice vibration modes of the constituent solid materials. These bands have a high diagnostic value because they are sensitive to chemical composition, mineralogy (crystallinity, modification), temperature, and size of the dust. However, they depend also on the shape and agglomeration of the grains. Particularly, the dust morphology directly affects the detected band position and profile (e.g., Tamanai et al. 2006a; Min et al. 2007; Takigawa et al. 2009).

Both experimentally measured and simulated spectra are employed for the interpretation of observed spectra. In general, theoretical models for calculating scattering, absorption, and extinction efficiencies by solid particles (e.g., Mie 1908; Purcell \& Pennypacker 1973; Bohren \& Huffman 1983; Draine 1988; Mackowski \& Mishchenko 1996; Min et al. 2006a) have been preferentially applied since one can easily modify the input parameters such as the grain size, its geometry, and its composition. However, simulating the whole set of possible particle conditions including also irregularity, heterogeneity, and agglomeration in an optical model is a complicated matter. The assumption of spherical grains or other highly symmetric shapes often leads to much too narrow band profiles, which do not at all match the observed spectra, for instance, in the case of crystalline silicate dust grains (e.g., Fabian et al. 2001). Instead of the single geometrical models, shape distribution models such as the continuous distribution of ellipsoids (CDE; Bohren \& Huffman 1983) or the distribution of hollow spheres (DHS; Min et al. 2005) have been introduced. The distribution functions of these models contain all the information with respect to the grain shape as well as the agglomeration, and 
are supposed to be selected in a way that they represent real grain ensembles (Mutschke et al. 2009). Nevertheless, knowledge about such distribution functions is still very limited. Although it has been demonstrated that shape factor distributions can be calculated from a discretization of the particle geometry (Min et al. 2006b), not all the particle characteristics (e.g., shape edges, contact geometry) can fully be taken into account due to the limited resolution of the discretization.

On the other hand, experimental spectroscopic IR absorption and/or transmission measurements of dust grains have been performed by making use of different preparation techniques such as pressing of pellets, thin film deposition, and adopting aerosol technology (e.g., Dorschner et al. 1977; Koike et al. 1980, 2006; Jäger et al. 1994, 2003; Mutschke et al. 1999; Posch et al. 2003; Tamanai et al. 2006a,b; Wetzel et al. 2013). After multiplication with Planck's function, the measured absorption coefficients may be directly compared to the astronomical emission spectra. Such measurements often naturally include the effect of a complicated particle morphology. In particular, dust grains are in most cases not well segregated mutually in nature, but rather are formed in the state of agglomerates, which strongly influences the optical properties of the respective media. Small particles $(\lesssim 1 \mu \mathrm{m})$ form agglomerates more easily than large ones ( $\gtrsim 1 \mu \mathrm{m})$ (Tamanai et al. 2009). The overall morphological effect (considering size, shape, and agglomerate state all together) on IR extinction spectra has been verified by aerosol spectroscopy (e.g., Tamanai et al. 2006a). However, the specific effects of agglomeration such as degree of porousness, size, and shape of agglomerates on IR band profiles are not well understood in detail because agglomerate structures are usually not sufficiently well defined in spectroscopic measurements. Experimental data that are focused on the influence of the agglomerate state have not been available up to now. Investigating the spectra of distinct well-defined agglomerate structures is therefore essential not only for understanding the effect of agglomeration on IR bands, but also for providing experimental results for further improvement of light scattering calculations.

In this paper, we demonstrate for the first time experimentally how the agglomerate structure and size affects the extinction spectra of amorphous $\mathrm{SiO}_{2}$ monosphere particles in the mid-IR region by the use of the engineering technique, Dedicated robotic manipulation (DRM) and IR microscopy at Synchrotron SOLEIL. The experimentally measured extinction spectra of agglomerates with well-defined morphological and structural characteristics are compared with theoretically calculated extinction spectra using different light scattering simulation methods.

\section{Experiment}

\subsection{Sample and sample preparation technique}

Monodisperse $\mathrm{SiO}_{2}$ particles (amorphous, manufactured by micromod) with nominal diameters of $1 \mu \mathrm{m}$ have been applied for the construction of well-defined particle agglomerates. These particles are systematically arranged on an IR-transparent substrate (silicon, Si (111) wafer with a high resistance; Siltronix; $1 \mathrm{~mm}$ in thickness) by means of the DRM technique. This technique allows a fabrication of the well-defined agglomerates inside a scanning electron microscope (SEM) coupled to a focused ion beam (FIB) instrument (TESCAN LYRA XMU) by employing a nano-manipulator (e.g., Zimmermann et al. 2015). We also produce agglomerates where the particles are slightly embedded $(300 \mathrm{~nm}$ depth) in the $\mathrm{Si}$ substrate to examine the
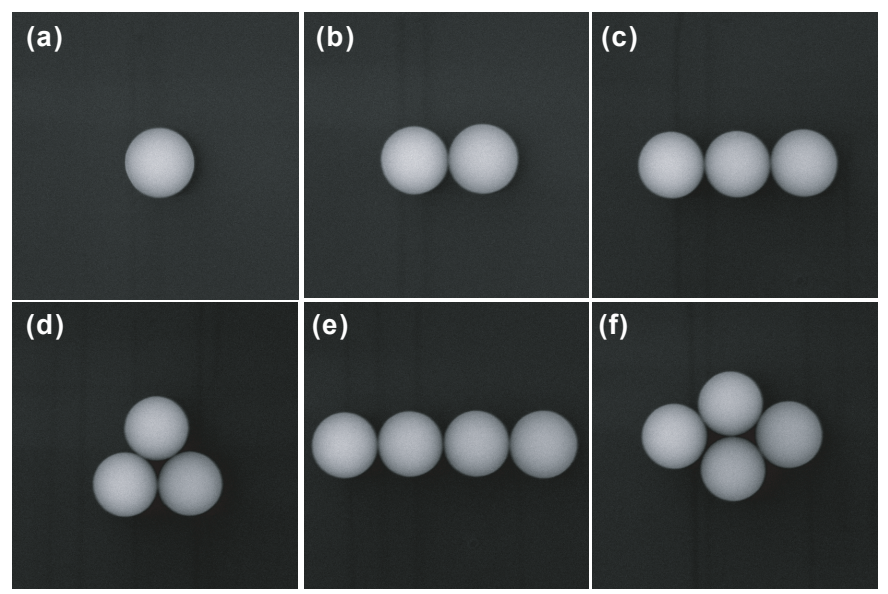

Fig. 1. SEM images of produced agglomerate structures: single sphere (panel $a$ ); two particles are linearly arranged ( 2 linear: panel $b$ ); three particles are linearly arranged (3 linear: panel $c$ ); three particles are arranged in a triangular shape (triangle: panel $d$ ); four particles are linearly arranged (4 linear: panel $e$ ); four particles are arranged in a diamond shape (diamond: panel $f$ ). The magnification of the images is $36 \mathrm{~K}$, and each sphere has a diameter of $1 \mu \mathrm{m}$. In panel $f$ a slight deviation from the ideal structure with perfect touching of all spheres in visible.

influence on different contact conditions of the substrate. Any substrate contacts are likely to change the electrical fields interacting with the particle (matrix effect). In order to minimize this effect, we focus on the arrangement "on substrate" but also evaluate its importance by a comparison with the spectra for slightly embedded particles.

The positioning of each particle is achieved via dedicated nano-manipulation techniques using piezoelectrically driven actuators and end effectors with an optimized geometry. The robotic sequence is monitored and controlled by means of insitu visual feedback provided by the SEM. Both the SEM with the FIB and the nano-manipulator are available at the University of Oldenburg (Division Microrobotics and Control Engineering, AMiR) together with their original software, and at the MaxPlanck Institute for the Science of Light where it is feasible to arrange particles systematically.

Agglomerate structures with particle numbers up to four (linear and compact configurations) have been fabricated. Representative SEM images are shown in Fig. 1. We prepared three sets of each agglomerate structure to enhance the reliability of the measurements. As parameters of interest in this study, we vary systematically the angle between "bonds" for agglomerates of three (linear and triangle) and four (linear and diamond) particles as well as the elongation of linear agglomerates (two to four particles).

\subsection{Measurement procedure}

IR microspectroscopic extinction measurements have been carried out at the French National Synchrotron Facility, SOLEIL. The synchrotron based IR beamline (SMIS) can be focused to sample spots down to the diffraction limit. This enables us to maintain a high beam intensity and thus spectra with a high signal to noise ratio even for sample structures smaller than the wavelength can be achieved. The Thermo Fisher NIC-PLAN IR microscope equipped with a liquid nitrogen cooled mercury cadmium telluride (MCT) detector is coupled to a NEXUS 5700 Fourier-transform infrared (FTIR) spectrometer. The aperture 


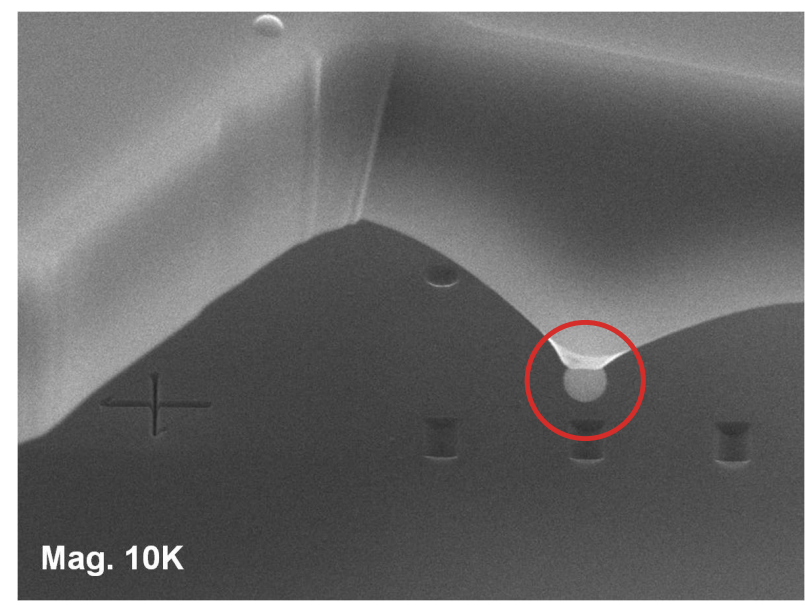

Fig. 2. SEM image (side view) of the particle arrangement process with a nano-manipulator, right before releasing an $\mathrm{SiO}_{2}$ particle $(d=1 \mu \mathrm{m}$, marked in red) from the tip of the manipulator to place it in a notch formed by the FIB at AMiR.

size has been set to $10 \times 10 \mu \mathrm{m}^{2}$. The measurements are performed in the wavelength range between 2.5 and $15 \mu \mathrm{m}$ at a resolution of $4 \mathrm{~cm}^{-1}$ with several 1000 scans per spectrum ${ }^{1}$. Measured spectra have been smoothed using the Savitzky-Golay algorithm.

Sample spectra have been measured at individual spheres and for five sets of agglomerates (see Sect. 2.1) with linearly polarized radiation for two different polarization directions (both parallel to the substrate). For this purpose, a wire grid polarizer with an extinction ratio for the two different polarization directions of about 750:1 (at $\lambda \sim 9 \mu \mathrm{m}$ ) was placed just above the objective lens in the IR microscope. In order to change the polarization direction by $90^{\circ}$, the sample was rotated, while the orientation of the polarizer remained fixed. This was necessary because the synchrotron IR beam is already linearly polarized to about $75 \%$. Consequently, the orientation of the polarizer was permanently aligned with the pre-polarization direction, which resulted in a polarization degree of better than $99.9 \%$. Contributions of the other polarization direction can therefore be neglected.

Because of the partial pre-polarization, we only show experimental spectra taken with the polarizer. Unpolarized spectra were calculated by averaging both polarization directions. Extinction spectra (decadic logarithm of the relative transmittance) were calculated from the ratio of sample and reference intensity spectra $\left(\right.$ Extinction $\left.=\log \left(I_{\text {sample }} / I_{\text {ref }}\right)\right)$. Significant extinction from the $\mathrm{Si}-\mathrm{O}-\mathrm{Si}$ antisymmetric stretching band appears around $9 \mu \mathrm{m}$. This band has the highest IR activity among the $\mathrm{SiO}_{2}$ phonon modes. Related to the strong IR activity of that mode, pronounced particle-phonon-polariton effects lead to clearly measurable shifts of the extinction maxima away from the phonon frequency of the bulk material. In this work we are interested in the agglomeration effects in extinction and thus restrict our discussion to the wavelength range between 7.5 and $10.5 \mu \mathrm{m}$

\subsection{Matrix effect}

When a particle is surrounded by a higher refractive index medium, its resonance frequency undergoes a redshift due to the

\footnotetext{
1 See more details at http://www.synchrotron-soleil.fr/ portal/page/portal/Recherche/LignesLumiere/SMIS
}

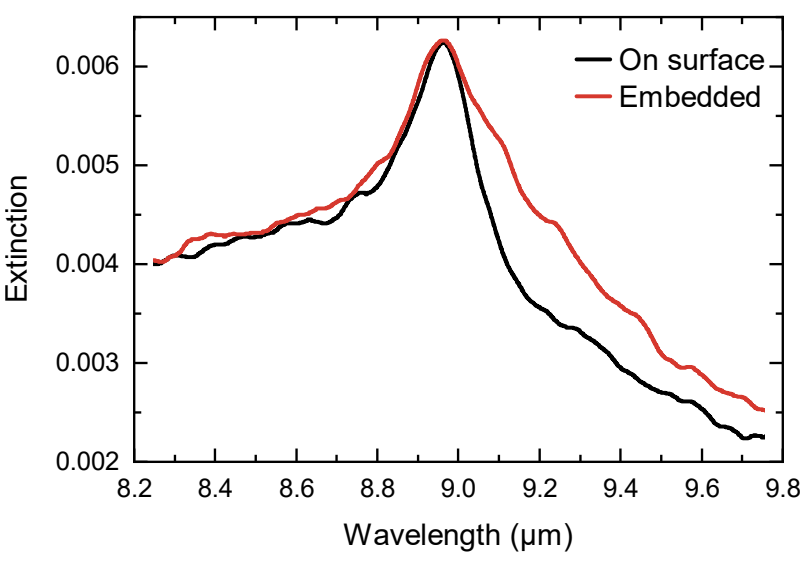

Fig. 3. Extinction spectra of two single particles (from the same batch) that are situated on the surface of a Si-substrate (black line) and embedded $300 \mathrm{~nm}$ in a Si-substrate (red line), respectively. The incidence of light was perpendicular to the substrate.

modified boundary conditions for its electromagnetic polarization (e.g., Tamanai et al. 2006a). The refractive index of highly resistive $\mathrm{Si}$ is approximately 3.4 in the $10 \mu \mathrm{m}$ wavelength region. Such Si does not possess any real part of the dynamic conductivity in the mid IR. In order to clarify the effect of the high refractive index of $\mathrm{Si}$ on the IR spectra of the agglomerates, we have prepared two types of samples where single particles are situated on or embedded in (ca. $300 \mathrm{~nm}$ in depth) the Si material. For the latter, nano-sized notches are formed by using the FIB instrument (a focused $\mathrm{Ga}$ beam) to accommodate the particles. An impression of the arrangement of $\mathrm{SiO}_{2}$ monosphere particles in such notches is shown in Fig. 2.

Figure 3 shows the extinction spectra of single particles on or embedded in the Si substrate. Only $20 \%$ of the particle volume is embedded in the Si substrate, but the matrix effect is clearly visible in the form of a shoulder of the $\mathrm{Si}-\mathrm{O}$ stretching vibration band towards longer wavelengths. The appearance of this shoulder may be interpreted as a broadening of the resonance due to a range of boundary conditions existing over the surface of the particle; a resonance peak shift, which would be expected for total embedding into the substrate, is not observed in these measurements. Similarly, the extinction strength is not influenced by the partial embedding.

Figure 4 shows the extinction spectrum for a single particle measured by IR microscopy and calculated by Mie theory. An ensemble spectrum measured by aerosol spectroscopy is also given for comparison. We note that the band width of the resonance band is quite similar for all spectra. The Mie calculation for the surrounding medium being air exhibits an even marginally broader band towards longer wavelengths than both the experimental results. Since the band broadening found for embedded particles is clearly not seen in the spectrum of the $\mathrm{SiO}_{2}$ particles on a $\mathrm{Si}$-substrate, we consider that the matrix effect is minimal in our measurements in the case of Si-supported $\mathrm{SiO}_{2}$ particles and that it is justified to neglect the effect of the underlying $\mathrm{Si}$ substrate. Therefore, the surrounding medium for some of the theoretical calculations has been set to be air for simplification. It should be mentioned that the particles used in the aerosol measurements and in the measurements shown in Fig. 3 have been taken from different $\mathrm{SiO}_{2}$ powder batches than those used in the further course of the paper. Therefore, the values of the peak positions cannot be compared directly with those listed in Table 1. 


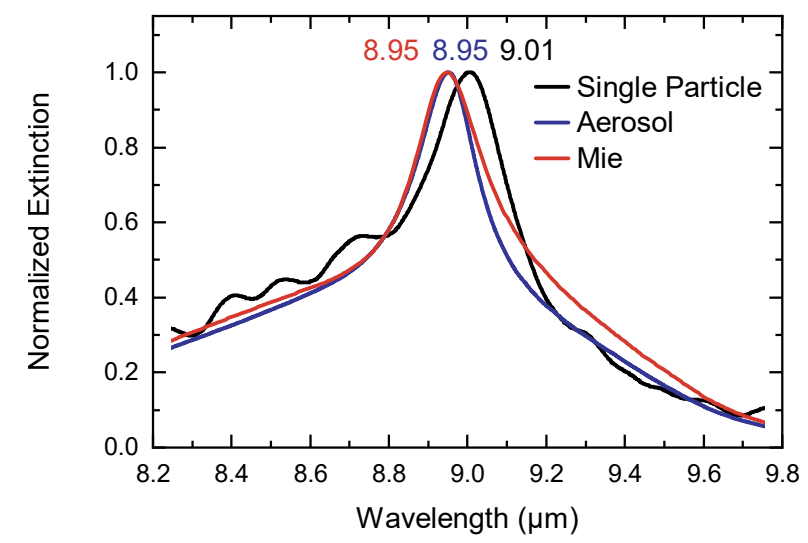

Fig. 4. Normalized extinction spectra obtained from IR microscopy for a single particle on an Si-substrate (black line), the Mie calculation (red line), and the aerosol spectroscopy (blue line).

Table 1. Peak positions of the IR bands measured in the two polarization directions for single spherical particles and for the five different agglomerate structures.

\begin{tabular}{lcc}
\hline \hline \multirow{2}{*}{ Agglomerate structure } & \multicolumn{2}{c}{ Peaks $(\mu \mathrm{m})$} \\
\cline { 2 - 3 } & $E_{\|}$ & $E_{\perp}$ \\
\hline Single & 9.01 & 9.01 \\
2 linear & 9.06 & 9.00 \\
3 linear & 9.08 & 9.00 \\
Triangle & 9.03 & 9.00 \\
4 linear & 9.12 & 8.97 \\
Diamond & 9.03 & 9.03 \\
\hline
\end{tabular}

\section{Theoretical approaches}

The experimentally measured extinction spectra are compared with theoretical simulations considering exactly the same particle shape, size, and agglomerate state as the arranged ones. The Mie theory is applied for a single particle (Mie 1908) whereas the discrete dipole approximation (DDA; e.g., Purcell \& Pennypacker 1973; Draine 1988), the finite-difference time-domain (FDTD; e.g., Yee 1966; Taflove \& Brodwin 1975; Sakamoto et al. 2014), and T-matrix methods (e.g., Waterman 1971; Mishchenko 1990; Mackowski \& Mishchenko 1996) are utilized for the agglomerates. The DDA and T-matrix method are both based on the surface numerical integration of the Maxwell equations for electromagnetic scattering. In contrast, the FDTD method discretizes the partial differential form of the Maxwell equations. Because the Si-wafer substrate underneath the $\mathrm{SiO}_{2}$ particles does not have a clear influence on the particle spectrum, the Si substrate is not taken into account in the DDA and T-matrix simulations for simplification.

In all simulations, we apply optical constants of amorphous $\mathrm{SiO}_{2}$ that have been derived by a Kramers-Kronig analysis (KKTRANS code by V. Ossenkopf ${ }^{2}$ ) of aerosol extinction spectra of similar $\mathrm{SiO}_{2}$ particles. The aerosol spectra in detail have been reported in Tamanai et al. (2006b). We note that these optical constants are not fully appropriate to reproduce the measured spectra because the optical properties of the various $\mathrm{SiO}_{2}$ powder samples varied, also because of different storage conditions. Wetzel (2012) demonstrated that the resonance frequency of evaporated $\mathrm{SiO}$ films shifted from 10.16 to $9.96 \mu \mathrm{m}$ due to the

\footnotetext{
2 https://hera.ph1.uni-koeln.de/ ossk/ftpspace/ fortran/
}

interaction of the precursor material with water from the ambient atmosphere. Our experiments are not done in a vacuum and so degradation effects cannot be avoided. However, in principle it is possible to determine the right optical data for the nanospheres and then to use them for simulations (e.g., Vogt et al. 2017). This only makes sense if all experiments are done immediately after each other, which was impossible in this study in which the nanomanipulation and the spectroscopy at the synchrotron had to be done at different, predefined dates. Therefore, we will not compare the calculated and measured spectra directly. Instead we will qualitatively compare and discuss the various spectra (Sect. 4.5).

The basic idea of the DDA method is that a solid particle is formed by an array of $N$ point dipoles in a lattice that has a spacing which is much smaller than the wavelength of the incident radiation (e.g., Draine \& Flatau 1994). The higher the dipole density, the more closely the particle configuration is reproduced and the more accurate and stable are the computed spectra. Andersen et al. (2006) have verified the stability of the amorphous $\mathrm{SiO}_{2}$ band profile calculated by DDA. Although the dipole number 4475 for a linear five structure (spherical particles) exhibited a secondary resonance around 9 micron, the profile has been steadied from the number 7792 up. On the basis of these facts, we have applied approximately 100 000-200 000 dipoles, depending on the number of particles, to represent each agglomerate structure in order to achieve sufficient accuracy for the DDA calculations. We use the publicly available DDSCAT 7.2 code $^{3}$.

The T-matrix method for a cluster of spheres is based on the superposition principle of the scattered field of a constituent particle. In the current paper, a constituent particle is spherical; therefore, the scattered field of each particle can be obtained by Mie theory. Multiple scattering of light inside the cluster is solved self-consistently by using the addition theorem for vector spherical wave functions (e.g., Mackowski 1991). This approach is exact to the truncation error of vector spherical wave function expansions. We use the publicly available Multiple Sphere T-Matrix (MSTM) code, version 3.0 ${ }^{4}$. We adopt the convergence criterion of $10^{-8}$ to estimate the maximum expansion order of the cluster T-matrix.

The FDTD method solves Maxwell's equations by discretizing a three-dimensional volume in a large number of cubic unit cells. Electric and magnetic field components are calculated alternately at discretized points in time by solving Maxwell's equations in each unit cell. The simulation volume was surrounded by perfectly matched layers (PML) absorbing incoming light without any reflection. The $\mathrm{SiO}_{2}$ spheres and aggregates on the silicon substrate are illuminated using a broadband plane wave. Scattering, absorption, and extinction crosssections have been calculated using the total-field scattered-field approach. The FDTD method takes advantage of all symmetries provided by the simulated structure in order to minimize the computational effort. Convergence tests have been performed in order to ensure accurate results.

\section{Spectroscopic results}

\subsection{Maximum intensity of extinction}

Figure 5 shows the extinction spectra of all six structures, measured in the region of the $\mathrm{Si}-\mathrm{O}$ stretching vibration band around $9 \mu \mathrm{m}$. To obtain unpolarized spectra, both polarization directions

\footnotetext{
3 Details at http://www.astro.princeton.edu/ draine/ index.html

4 See details in http://www.eng.auburn.edu/ dmckwski/ scatcodes/
} 


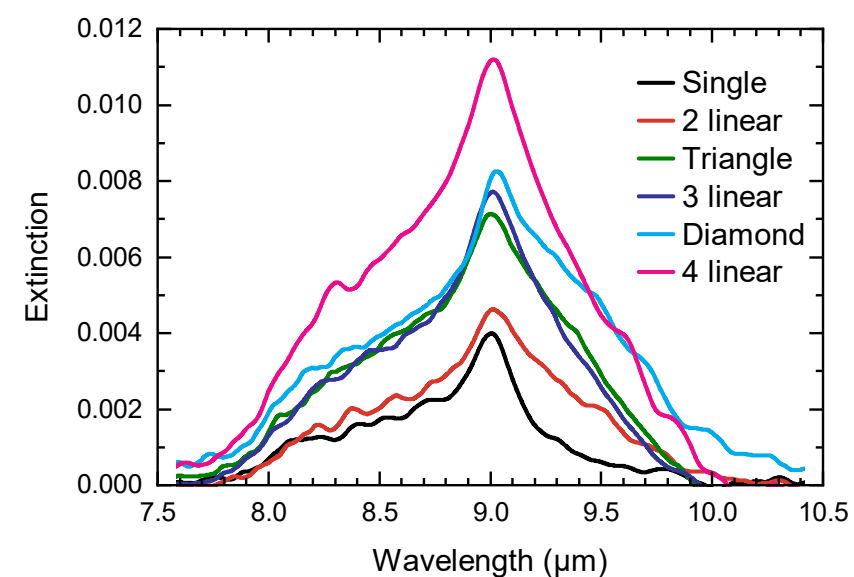

Fig. 5. Experimentally determined extinction spectra of the six different agglomerate structures, calculated by averaging parallel and perpendicular polarized spectra.

were measured individually and averaged afterwards. As could be seen in that figure, the extinction strengths gradually increase with increasing the number of particles, as expected. For the diamond agglomerate, the intensity appears actually to be somewhat too low, which is due to a relatively small intensity in the measurement with parallel polarization. This is likely a result of an imperfect centering of the microscope beam on the agglomerate in that measurement. The spectral band shape is almost not influenced by this error because the band has almost identical profiles in the two polarizations (see below). However, we take this as a reason to show only normalized band profiles in the further course of the paper.

\subsection{Orientation effects}

Figure 6 represents the normalized extinction spectra measured with a polarizer inserted into the beam and oriented in a way that the electric field is either parallel ( $E_{\|}: p$-polarized) or perpendic$\operatorname{ular}\left(E_{\perp}: s\right.$-polarized $)$ to the major axes of the respective agglomerates, as depicted in the inserted sketches. For comparison, the respective spectrum of the single particle is shown in each plot.

The spectra in Fig. 6 show strong deviations in the band profiles depending on polarization and on the type of agglomerate. For all agglomerates, the peak positions are redshifted for $E_{\|}$and marginally blueshifted for $E_{\perp}$ compared to the single particle, except for the diamond shape where both resonances are redshifted (Table 1). In all cases, the perpendicular polarized peak is close to that of the single sphere. The band broadening towards longer wavelengths is seen in all of the spectra measured in parallel polarization and for the non-linear agglomerates also in perpendicular polarization. Hence, a remarkable disparity between the extinction spectra of $E_{\|}$and $E_{\perp}$ directions is not observable for the triangle and diamond cases in comparison with the linearly arranged structures.

For the linear agglomerates, the perpendicularly polarized spectra are not broadened towards longer wavelengths but resemble closely the spectra of the single sphere in that respect. However, for the 4 linear agglomerate, a clear extinction enhancement on the lower wavelength side can be seen (Fig. 6d).

\subsection{Theoretically calculated extinction spectra: 4 linear and diamond}

The theoretical models DDA, FDTD, and T-matrix have been applied for the prediction of the extinction band profiles for all fabricated agglomerate structures. First, we select the extreme cases of the elongated (4 linear) and close-packed (diamond) agglomerate structures here for the discussion of the results. Figures $7 \mathrm{a}, \mathrm{c}$ and e represent the extinction spectra in the wavelength range between 8.4 and $9.8 \mu \mathrm{m}$ calculated by the DDA, T-matrix, and FDTD methods, respectively, for four linearly arranged particles, together with the result of the Mie theory for a single sphere. Similarly, the calculated extinction spectra obtained from the diamond agglomerate structure are shown in Fig. 7b (DDA), d (T-matrix), and f (FDTD).

In the case of the 4 linear structure, all theoretically calculated spectra at $E_{\|}$polarization exhibit very broad peaks (a nearly trapezoidal profile) in the wavelength range between 9.1 and $9.4 \mu \mathrm{m}$. Among these spectra, the one produced by the T-matrix calculation is slightly less broadened. On the other hand, the extinction spectra at $E_{\perp}$ polarization calculated by DDA, T-matrix, and FDTD are identical to each other and very similar to the band profile of the Mie calculated spectrum, apart from a slight blueshift of the peak and a slight increase of the extinction in the short-wavelength wing $(8.4-8.7 \mu \mathrm{m})$. The unpolarized spectra are averages of the polarized spectra (assuming the same amplitudes for each direction) and show a pronounced broadening beyond the peak position towards longer wavelengths, which is related to the contribution of the parallel polarization. The peak position is here slightly redshifted compared to the Mie spectrum. For the parallel polarization, a peak position is difficult to define, but would be located beyond $9.1 \mu \mathrm{m}$ for all theoretical spectra.

With regard to the diamond agglomerate structure, the extinction spectra calculated by DDA and FDTD methods are again very much alike, while the shoulder-like feature beyond the resonant peak toward longer wavelengths is less pronounced in the result of the T-matrix method. Consequently, the bandwidth of the extinction spectrum obtained from the T-matrix calculation is slightly narrower than for the other two calculated results as shown in Figs. 7b,d and f. Unlike in the case of the 4 linear agglomerate, the band is broadened in both the polarized spectra, as expected. The fact that the broadening is stronger for the perpendicular than for the parallel polarization is consistent with the elongation of the agglomerate in the respective direction. Correspondingly, the peaks of the band profiles are located at shorter wavelengths for $E_{\|}$polarization than for $E_{\perp}$.

\subsection{Theoretically calculated extinction spectra: 2 linear, 3 linear, and triangle}

We show here the calculated spectra for the remaining agglomerate structures, which consist of two and three particles. As mentioned in Sect. 4.3, the extinction spectra calculated by the FDTD and DDA methods are almost identical. Therefore we restrict the spectra shown in Fig. 8 to the results of the DDA and T-matrix calculations for both the unpolarized and polarized cases of incident radiation. In addition, we compare them to the Mie calculation (single sphere in air) result.

By comparison of the spectra in Fig. 8(a-d) with Fig. 7(a,c), we observe a clear trend with increasing length of the linear agglomerates for the above-discussed agglomeration effects, namely the redshift and broadening of the band in $E_{\|}$polarization. These effects are already significant for the 2 linear agglomerate. The spectrum in $E_{\perp}$ polarization remains at the position of the Mie spectrum with only a slight increase of the blue shoulder. The results of the T-matrix calculations show that the effects are again weaker than the DDA results. 

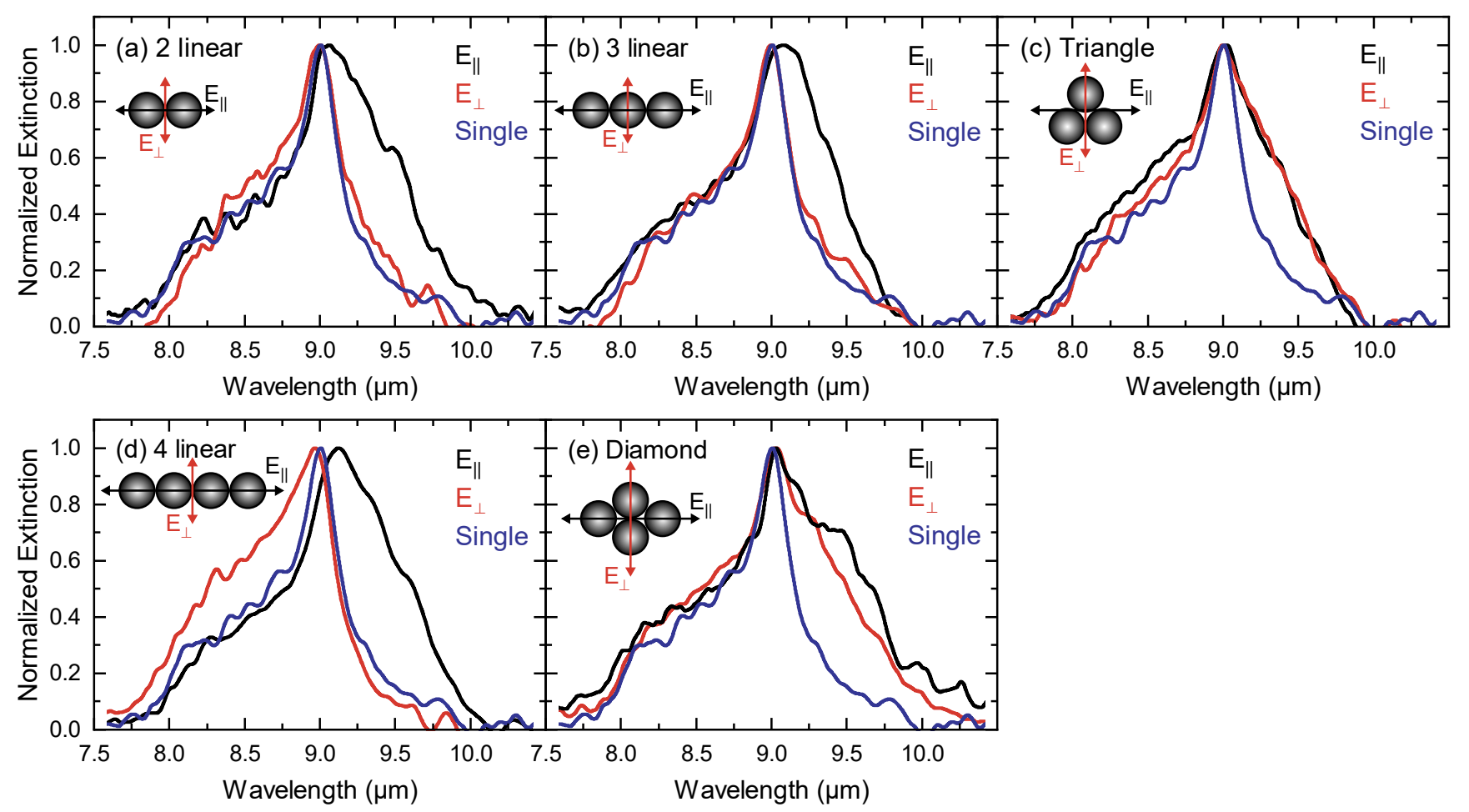

Fig. 6. Normalized extinction spectra using polarized light ( $E_{\|}$and $E_{\perp}$ directions) measured for the five different agglomerate structures together with the extinction spectra of a single particle: 2 linear (panel a); 3 linear (panel b); triangle (panel c); 4 linear (panel d); diamond (panel e). The spectra are normalized to the maximum extinction.

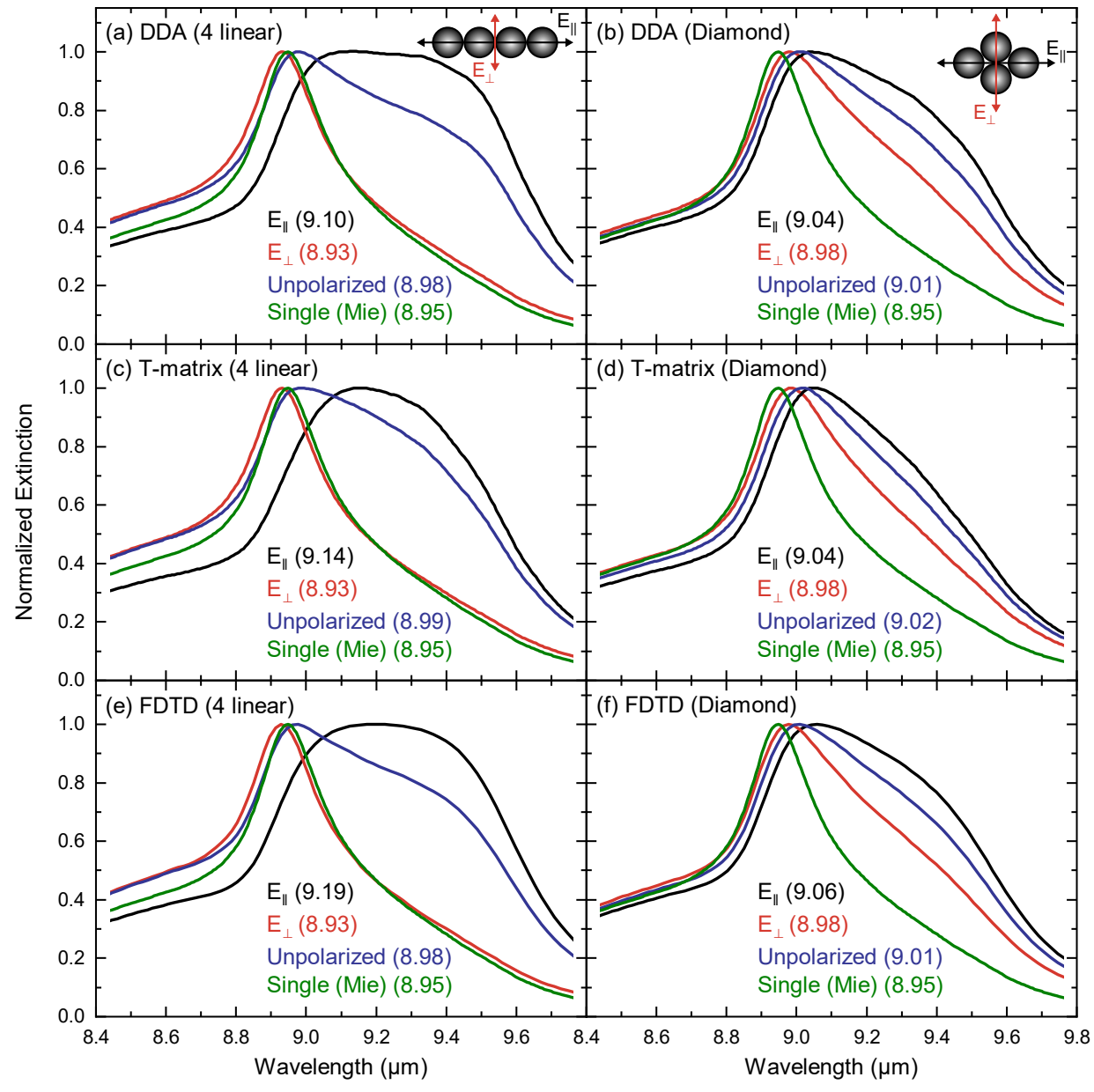

Fig. 7. Normalized extinction spectra of two different agglomerate structures (4 linear and diamond) obtained from the three different theoretical methods: DDA results (panels $a, b$ ); T-matrix results (panels $c, d$ ); FDTD results (panels e,f). The unpolarized spectra represent averages of the two polarization directions. The result of Mie theory for single spheres is shown for comparison. The values in parentheses indicate the peak positions $(\mu \mathrm{m})$. 


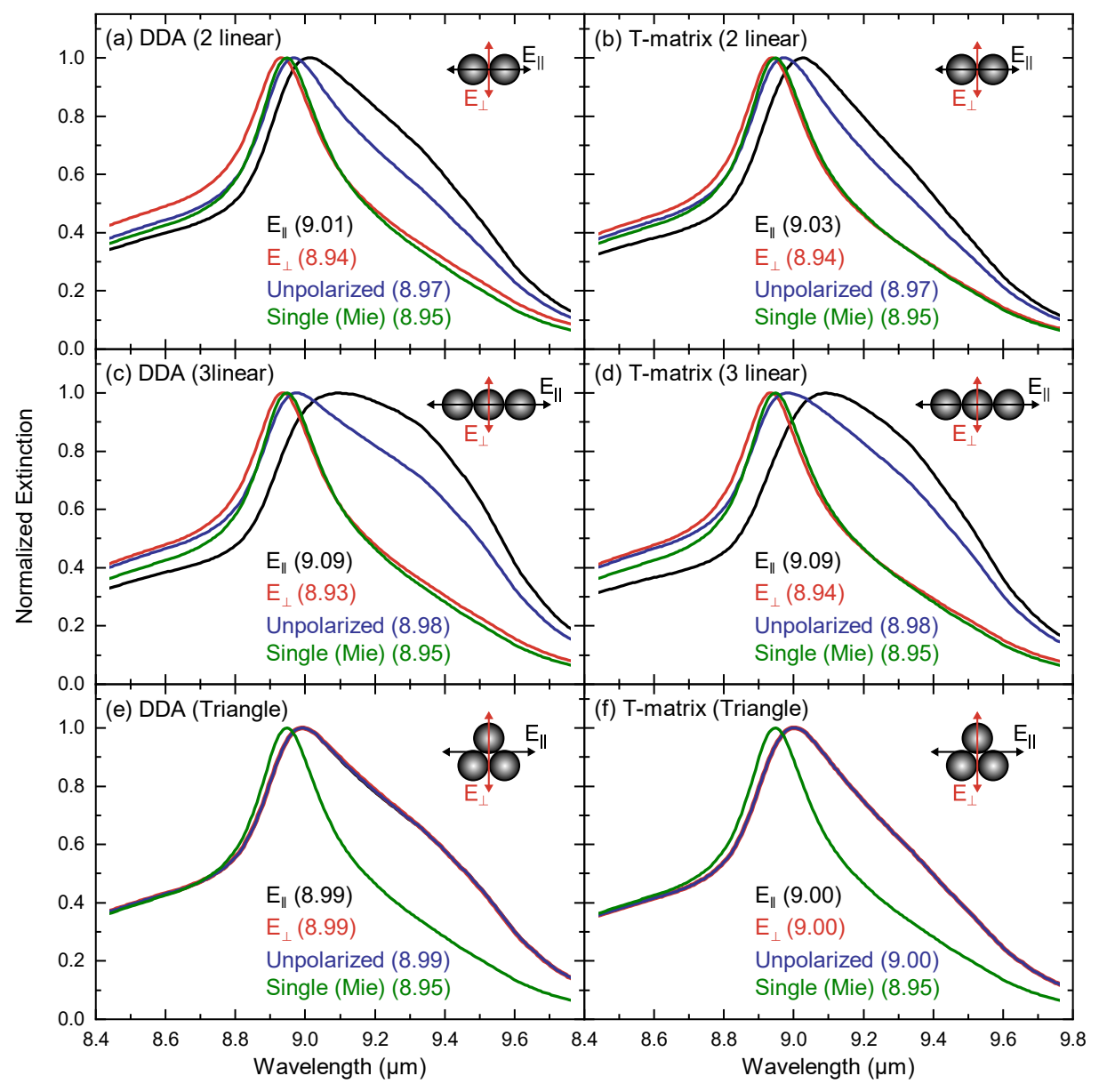

Fig. 8. Normalized extinction spectra of the remaining three agglomerate structures ( 2 linear, 3 linear, and triangle) obtained with the DDA and T-matrix methods: 2 linear structure (panels $a, b)$; 3 linear structure (panels $c, d$ ); triangle structure (panels $e, f$ ). The unpolarized spectra represent averages of the two polarization directions. The result of Mie theory for single spheres is shown for comparison. Note: the values in parentheses indicate the peak positions $(\mu \mathrm{m})$.
The calculated spectra for the triangle structure (Figs. 8e and f) are exceptional in the fact that both polarization directions produce exactly the same band profile. Moreover, this reproduces qualitatively the experimental finding (Fig. 7c). Although the shoulders beyond the resonance peak are slightly weaker in the experimentally measured spectra than in both the results of the DDA and T-matrix calculations, they appear to be equally strong in both polarization directions as well. This finding corresponds to the symmetry of the triangular structure.

\subsection{Comparison of measured and simulated spectra}

From the previous discussions, it is evident, that the major effects of agglomeration have been detected in both the measured and calculated spectra of the $\mathrm{Si}-\mathrm{O}$ stretching vibration band. The peak shifts to the red in $E_{\|}$and to the blue in $E_{\perp}$ polarization, respectively, as predicted by the models for linear agglomerates, are also seen in the measured spectra, particularly for the longest agglomeration chain (4 linear). The band broadening of the measured spectra for $E_{\|}$polarization is not as strong as predicted by the calculations where the trapezoidal-shaped peak extends up to about $9.5 \mu \mathrm{m}$ depending on the simulation method. However, very likely this cannot be seen as a criterion to judge between the different models, as we noted already that the optical constants used can be responsible for quantitative differences between simulated and measured spectra. For the agglomerates, experimental spectra might deviate also because of the imperfect touching of neighbor spheres. The slight enhancement in the blue wing for the $E_{\perp}$ profiles predicted for the linear agglomerates, however, is seen as an even more significant effect in the spectrum of the 4 linear agglomerate (Fig. 6d), while it is too small compared to the noise in the spectra for the shorter agglomerate chains.

For the close-packed agglomerates such as the diamond structure, the measured spectrum agrees well with the calculated ones regarding the band broadening and peak shift to longer wavelengths for both the polarization directions. The broadening as predicted by the simulations is stronger for the polarization along the longer axis, which is apparently seen in the measured spectra as well, but with a high degree of uncertainty.

\section{Conclusion}

In this paper IR microspectroscopic extinction measurements of well-defined agglomerates composed of up to four amorphous monosphere $\mathrm{SiO}_{2}$ particles have been studied in the wavelength range between 7.5 and $10.5 \mu \mathrm{m}$ for two different polarizations. The experimental spectra taken at the French National Synchrotron Facility, SOLEIL, are compared with the results of light scattering simulations done with three different methods (DDA, T-matrix, FDTD). In this way, we could verify the dependence of the extinction spectrum in the range of the $\mathrm{Si}-\mathrm{O}$ stretching band on the agglomerate configurations. We summarize the results as follows:

- As the number of particles increases, the extinction strength increases as expected. The broadening of the $\mathrm{Si}-\mathrm{O}$ stretching band toward longer wavelengths is clearly detected for all agglomerate structures with light that contains electric field components parallel to an axis in which the particles are agglomerated. The broadening tends to become also more 
prominent with increasing number of particles (e.g., for the linear agglomerates); however, different agglomerate structures with the same number of particles show distinct band broadening.

- While the broadening toward longer wavelengths is associated with $E_{\|}$-polarized light, nearly a single-particle band profile is obtained with $E_{\perp}$-polarized light for the linearly arranged particles.

- In the case of close-packed triangle, the band profiles measured from the $E_{\|}$and $E_{\perp}$ cases resemble each other and so does the unpolarized profile. Compared to the single sphere, spectral broadening on the red side is obvious and indicates the coupling effect to neighboring spheres.

- The FDTD and DDA results are very much like each other in most cases whereas the T-matrix spectra show slightly narrower band profiles for the larger aggregates.

Our results show for small agglomerates how the arrangement of the dust grains has a great impact on IR extinction spectra in the range of a strongly IR active vibrational mode, and that FDTD, DDA, and T-matrix simulations describe the measured spectra well. For the accuracy of the simulations, it is important to use optical data derived from proper material and a suitable grid resolution. In this way, it should be possible to clarify the conditions of dust grains, for example, whether grain growth takes place or not, or whether submicron-sized single grains, close-packed, elongated, or fluffy agglomerates composed of micron-sized grains dominate, via the detailed analysis of the shape and maximum of extinction spectra obtained from larger agglomerates.

Acknowledgements. We are grateful to C. Sand and P. Dumas for their technica supports at the French National Synchrotron Facility, SOLEIL, and to the referee, Dr. Joseph A. Nuth, for his valuable comments on the manuscript. Our project has been supported by the "Deutsche Forschungsgemeinschaft (DFG)" under the Priority Program SPP1385.

\section{References}

Andersen, A., Mutschke, H., Posch, T., Min, M., \& Tamanai, A. 2006, J. Quant. Spectr. Rad. Transf., 100, 4
Bohren, C. F., \& Huffman, D. R. 1983, Absorption and Scattering of Light by Small Particles (New York: John Wiley \& Sons)

Dorschner, J., Friedemann, C., \& Gürtler, J. 1977, Ap\&SS, 48, 305

Draine, B. T. 1988, ApJ, 333, 848

Draine, B. T., \& Flatau, P. J. 1994, J. Opt. Soc. Am. A, 11, 1491

Fabian, D., Henning, T., Jäger, C., et al. 2001, A\&A, 378, 228

Jäger, C., Mutschke, H., Begemann, B., Dorschner, J., \& Henning, T. 1994, A\&A, 292, 641

Jäger, C., Dorschner, J., Mutschke, H., Posch, T., \& Henning, T. 2003, A\&A, 408, 193

Koike, C., Hasegawa, H., \& Manabe, A. 1980, Ap\&SS, 67, 495

Koike, C., Mutschke, H., Suto, H., et al. 2006, A\&A, 449, 583

Mackowski, D. W. 1991, Roy. Soc. London Phil. Trans. Ser. A, 433, 599

Mackowski, D. W., \& Mishchenko, M. I. 1996, J. Opt. Soc. Am. A., 13, 2266

Mie, G. 1908, Ann. Phys., 25, 377

Min, M., Hovenier, J. W., \& de Koter, A. 2005, A\&A, 432, 909

Min, M., Dominik, C., Hovenier, J. W., de Koter, A., \& Waters, L. B. F. M. 2006a, A\&A, 445, 1005

Min, M., Hovenier, J. W., Dominik, C., de Koter, A., \& Yurkin, M. A. 2006b, J. Quant. Spectr. Rad. Transf., 97, 161

Min, M., Waters, L. B. F. M., de Koter, A., et al. 2007, A\&A, 462, 667

Mishchenko, M. I. 1990, Ap\&SS, 164, 1

Mutschke, H., Andersen, A., Clément, D., Henning, T., \& Peiter, G. 1999, A\&A, 345,187

Mutschke, H., Min, M., \& Tamanai, A. 2009, A\&A, 504, 875

Posch, T., Kerschbaum, F., Fabian, D., et al. 2003, ApJS, 149, 437

Purcell, E. M., \& Pennypacker, C. R. 1973, ApJ, 186, 705

Sakamoto, S., Tsuru, H., Toyada, M., \& Asakura, T. 2013, in Computational Simulation in Architechtrural and Environmental Acoustics: Methods and Applications of Wave-Based Computation, eds. T. Sakuma, S. Sakamoto, \& T. Otsuru (Tokyo: Springer), 11

Taflove, A., \& Brodwin, M. E. 1975, IEEE Trans. Microw. Theory, 23, 623

Takigawa, A., Tachibana, S., Nagahara, H., Ozawa, K., \& Yokoyama, M. 2009, ApJ, 707, L97

Tamanai, A., Mutschke, H., Blum, J., \& Meeus, G. 2006a, ApJ, 648, L147

Tamanai, A., Mutschke, H., Blum, J., \& Neuhäuser, R. 2006b, J. Quant. Spectr. Rad. Transf., 100, 373

Tamanai, A., Mutschke, H., Blum, J., et al. 2009, A\&A, 501, 251

Vogt, J., Zimmermann, S., Huck, C., et al. 2017, ACS Photonics, 4, 560

Waterman, P. C. 1971, Phys. Rev. D, 3, 825

Wetzel, S. 1966, PhD Thesis, Heidelberg University, Germany

Wetzel, S., Klevenz, M., Gail, H.-P., Pucci, A., \& Trieloff, M. 2013, A\&A, 553, A92

Yee, K. S. 1966, IEEE Trans. Antennas Propag., 14, 302

Zimmermann, S., Tiemerding, T., \& Fatikow, S. 2015, IEEE-ASME Trans. Mech., 20, 2031 